\title{
Functional Gene Trap Mutagenesis Screen for Genes and Molecular Mechanisms Underlying Amyloid- $\beta$-induced Inflammasome-mediated Neuronal Death
}

Jeremy Kean Yi Yap

International Medical University

Benjamin Simon Pickard

University of Strathclyde

Sook Yee Gan

International Medical University

Elaine Wan Ling Chan ( $\nabla$ elainechan@imu.edu.my )

International Medical University https://orcid.org/0000-0002-8627-0161

\section{Research article}

Keywords: Alzheimer's disease, neuroinflammation, NLRP1 inflammasome, gene trap mutagenesis, mitochondria dysfunction, TGF- $\beta$ signalling, cytoskeletal dynamics

Posted Date: June 4th, 2020

DOI: https://doi.org/10.21203/rs.3.rs-19699/v1

License: (9) This work is licensed under a Creative Commons Attribution 4.0 International License.

Read Full License 


\section{Abstract \\ Background}

Neuroinflammation is increasingly recognized for its roles in AD pathogenesis which, in part, links amyloid-beta $(A \beta)$ to neuronal death. While commonly associated with glial cells, neurons themselves are able to participate in neuroinflammation signalling, potentially leading to widespread neuronal suicide. The presence of the inflammasomes such as NLRP1 in neurons accelerates A $\beta$-induced neuroinflammation and has been shown to trigger neuronal pyroptosis in murine AD models. However, the pathways involved in $A \beta$ activation of inflammasomes has yet to be elucidated, especially in humans. In this study, we utilized a gene trap mutagenesis phenotypic screen approach to uncover the genes and biological pathways involved in inflammasome signalling in neurons and how it contributed to $A \beta-$ induced neuronal death.

\section{Results}

$A \beta$ significantly accelerated neuroinflammatory cell death in the presence of primed inflammasome. The gene trap mutagenesis screen discovered genes related to mitochondria function and TGF- $\beta$ signalling as significant contributors to $A \beta$-induced inflammasome-driven neuronal death. Additionally, genes associated with cytoskeletal reorganization were found to confer neuroprotection.

\section{Conclusion}

Our data presents a list of potentially important components of inflammasome signalling in neurons which makes promising therapeutic targets for future drug development against neuroinflammation in AD.

\section{Background}

Alzheimer's disease (AD) is a neurodegenerative disease which progressively interferes with behaviour, cognition, and memory, ultimately leading to death. It is the most common form of dementia, accounting for approximately $60-80 \%$ of total dementia cases. The estimated prevalence of $A D$ is $10-30 \%$ in populations above 65 years old [1]. Current AD therapies are primarily limited to symptom management and improvement of the quality of life of patients. As such, the race for the discovery of novel therapeutic targets for $A D$ is now more crucial than ever. Recently, studies on the innate immunity within the central nervous system (CNS) is beginning to gain traction as neuroinflammation becomes widely suspected as the missing link between the two pathological hallmarks of $A D$ (amyloid- $\beta(A \beta)$ and neurofibrillary tangles) and neuronal death [2]. In particular, the regulatory molecules of inflammation known as inflammasomes could be responsible for neuroinflammation in $A D$ as they enable an immune response against $A D$ pathologies in both microglia and in neurons $[3,4]$. The activation of inflammasomes results 
in caspase-1 mediated pyroptosis, a form of autolytic cell death characterised by the rupture of cell membrane followed by the release of pro-inflammatory cytokines [5]. The roles of inflammasomes, especially NLRP3, in AD has been widely implicates in glial cells, but not in neurons [6]. However, if neurons are able to participate in inflammasome-mediated neuroinflammation, it could lead to widespread neuronal pyroptosis, resulting in permanent neuronal loss. $A \beta$ has also been shown to activate the NIrp1 inflammasome leading to neuronal pyroptosis in murine models [7]. However, the precise mechanism of inflammasome activation by $A \beta$ in neurons is currently unclear. Given that the main constituent inflammasome in human neurons is NLRP1 but not NLRP3 [8, 9], we utilize a gene trap mutagenesis phenotypic screen approach to investigate the molecular signalling pathways involved in $A \beta$ induction of the NLRP1 inflammasome in a human neuron model.

Gene trap mutagenesis generates random insertional mutations via genomic integration of a gene trap plasmid vector. Originally used to generate mutant embryonic stem cells, gene trap mutagenesis has since undergone substantial development and found its usefulness in identification of novel gene functions and molecular mechanisms [10-12]. In this study, we employed pGTIV3, an advanced poly(A) trap vector containing a human $\beta$-actin promoter upstream of neoR gene and a rabbit $\beta$-globin-derived splice donor (SD) sequence [13]. A unique feature of this vector is the inclusion of a synthetic intron with a cis-acting mRNA destabilizing AU rich element (ARE) which degrades the transcribed Neomycin resistance (neoR) mRNA if expressed. In the event that the gene trap vector non-productively integrates into the genome outside of a gene, the synthetic intron is not removed resulting in the inclusion of the ARE which disintegrates the resulting neoR mRNA transcript and the cell does not gain resistance to neomycin selection. Only true SD splicing events resulting from disruptive vector insertion into a gene intron permit neomycin resistance under selection.

We introduced pGTIV3 into the SH-SY5Y neuroblastoma cell line genome to produce a library of cells which collectively represented approximately 15,000 different heterozygote gene mutations. In order to elucidate the biological pathways mediating $A \beta$-induced, inflammasome mediated neuronal death, we subjected the library cells to treatment with $A \beta$ and/or muramyl dipeptide (MDP). MDP is a derivative of bacterial peptidoglycan which is used as an activating ligand for the NLRP1 inflammasome [14]. In addition of stimulating inflammasome oligomerisation, MDP encourages the formation of NOD2-NLRP1 complex to induce NLRP1-dependent caspase-1 processing [15]. The treatment-recovery selection process is repeated over 7 cycles. Rapid amplification of CDNA ends (RACE), followed by targeted nextgeneration sequencing (NGS) were then performed to identify trapped genes. The identified genes represent significant regulators of the susceptibility of neurons to $A \beta$-induced inflammasome-mediated neuronal death.

\section{Results}

\section{MDP-primed inflammasome accelerates Aß-induced SH- SY5Y cell death}


Interestingly, when SH-SY5Y cells were exposed to $A \beta$, cell viability appeared to increase above the control level from the 4-hour time point and reached a peak at 18 hours, after which the cell viability sharply and steadily declined (Fig. 1). The viability of cells treated with $A \beta$ and $A \beta+$ MDP fell below $100 \%$ after 36 hours with $A \beta+$ MDP cells dying at a comparatively higher rate. Cells treated with MDP alone, however, showed no significant cell death throughout the duration of the assay. At the 72-hour time point, the viability of cells treated with $A \beta$ dropped to $72 \%$. Importantly, co-exposure of $A \beta$ with MDP could further decrease cell viability to $56 \%$, implying that $A \beta$ and MDP-primed inflammasome functioned in synergy to promote neuroinflammatory cell death. The trend of cell viability post-treatment was supported by microscopic images (Fig. 2). At 24 hours, the morphology of SH-SY5Y cells were similar across the groups. However, at 48 hours, cell lesions began to emerge, signifying the progress of cell death. Finally, at 72 hours, cells subjected to $A \beta$ and $A \beta+$ MDP treatments exhibited significant cell lesions compared to control and MDP treatment only. In order to maximise selection pressure in the gene trap mutagenesis screen, the duration of treatments at which the cell viability was at its lowest was selected. As such, the cells were exposed to the respective treatments for 72 hours per cycle.

\section{$A \beta$ and MDP synergistically intensified NLRP1 activity in SH-SY5Y cells}

In order to examine the effect of A 3 on the activity of NLRP1 inflammasome, the levels of NLRP1, inactive procaspase -1 and the P20 fragment of activated caspase- 1 were measured after exposure of SH-SY5Y cells to the respective treatments for 2 hours. The activation of inflammasomes simultaneously triggers autophagic degradation of the inflammasome complex as a negative regulatory mechanism to limit inflammation [20, 21]. As such, NLRP1 and caspase-1 levels must be detected prior to their degradation. As shown in Fig. 3, A $\beta$ by itself did not significantly affect the expression of NLRP1. Interestingly, however, $A \beta$ treatment did induce caspase-1 activation and cleavage, as shown by a 2-fold increase in P20 fragments compared to control. MDP was able to induce a significant increase in intracellular NLRP1 levels. At the same time, MDP was also generate significant expression and activation of caspase-1. However, a significant portion of caspase-1 remained in its inactive form. We then studied the capability of $A \beta$ to exacerbate neuroinflammation in the presence of MDP-primed NLRP1 activation. When cells were co-treated with MDP and $A \beta$, there was a remarkable surge in conversion of procaspase- 1 into its activated form, as indicated by a 5.6-fold in P20 fragment generation compared to control.

\section{Gene trap mutagenesis screen for signalling intermediates underlying $A \beta$-induced inflammasome-mediated neuronal death}

Through a gene allele representation analysis, 13,605 mutated genes were identified when the False Discovery Rate (FDR) cut-off was set to 1.0 (all sequenced genes regardless of their differential representation level significance), which denotes the number of genes successfully targeted by the gene trap vector in the parent library. When an FDR cut-off of 0.05 was applied, seven mutated genes were 
returned with a significant allele representation of $\log _{2}$ fold change ( $\log$ FC) more than 3 or less than -3 in one or more treatment groups (Table 1). The genes identified by the functional gene trap mutagenesis screen are considered to be important regulators of cell survivability under the different treatment conditions. It should be noted that the limma-voom software employed in this study is designed to compute differential gene expression, which we measured as a proxy for read depth as the trapped genes were expressed at a constant rate driven by the gene trap vector's built-in $\beta$-actin promoter. The differences in read depth reflects the changes in cell fitness caused by each trapped gene. The higher the read depth for a particular gene, the greater number of cells in the surviving population carrying its mutation, and vice versa.

The mutation of a susceptibility gene would promote the survival of the mutant containing cells, hence reflected in sequence read depth with positive log FC (Table 1). Henceforth, these genes will be referred to as 'susceptibility genes', reflecting their 'wild type' susceptibility effect. As shown in Table 1, three susceptibility genes (RHOT1, TGFBR1 and BEND5) were identified with log $\mathrm{FC}>3$ (reduction of gene function lead to $>8$ time more read depth in the treatment groups compared to control). The most significant susceptibility gene in $A \beta+$ MDP group was $R H O T 1$, as evidenced by its high gene trap representation (mutant gene $\log F C=7.11$ ). This was followed by $T G F B R 1$ which confers susceptibility in almost equal capacity in all three treatment groups, with mutant gene log $\mathrm{FC}$ values of 3.29, 3.39 and 3.17 for $A \beta, M D P$ and $A \beta+$ MDP respectively (Table 1, Fig. 4). Interestingly, BEND5 confers susceptibility when subjected to treatment with MDP only (mutant gene log $F C=4.06$ ) but not in the presence of $A \beta$.

Conversely, mutation of a naturally protective gene would result in reduced mutant cell survival, hence a read depth with negative log FC (Table 1). As shown in Table 1, four protective genes (CNP, SNW1, GREB1 and $S R G A P 3$ ) were identified with log $\mathrm{FC}<-3$ (reduction of gene function led to $>8$ time less read depth in the treatment groups compared to control). The most significant protective gene observed was $C N P$ (mutant gene $\log F C=-7.15$ ), which is the only protective gene recorded in the A + MDP group. SNW1 is a common protective gene in $A \beta$ and MDP groups, both with similar $\log F C(\log F C=-3.08$ and -3.34 respectively). However, $S R G A P 3$ is a protective gene unique to the $A \beta$ group (mutant gene log $F C=-4.82$ ), while GREB1 is unique to MDP group (mutant gene log $\mathrm{FC}=-3.73$ ). 
Table 1

List of genes with significant changes in gene trap representation relative to control. Significant 'wild type' susceptibility genes (log FC >3) and 'wild-type' protective genes $(\log \mathrm{FC}<-3)$ are bolded.

\begin{tabular}{|llllll|}
\hline Gene name & FDR & P-value & \multicolumn{2}{l}{ Log FC } \\
\cline { 4 - 6 } & & & A $\beta$ & MDP & A $\beta+$ MDP \\
\hline RHOT1 & $3.14 \times 10^{-3}$ & $2.31 \times 10^{-7}$ & -0.11 & 0.11 & 7.11 \\
\hline CNP & 0.02 & $2.73 \times 10^{-6}$ & -0.01 & 0.09 & -7.15 \\
\hline TGFBR1 & 0.02 & $4.088 \times 10^{-6}$ & 3.29 & 3.39 & 3.17 \\
\hline SNW1 & 0.03 & $8.88 \times 10^{-6}$ & -3.08 & -3.34 & -0.11 \\
\hline BEND5 & 0.04 & $1.83 \times 10^{-5}$ & -1.97 & 4.06 & -0.81 \\
\hline GREB1 & 0.04 & $1.95 \times 10^{-5}$ & 0.43 & -3.73 & 0.33 \\
\hline SRGAP3 & 0.04 & $2.16 \times 10^{-5}$ & -4.82 & -0.67 & 0.62 \\
\hline
\end{tabular}

\section{Discussion}

$A \beta$ is one of the major neurotoxic factors responsible for neurodegeneration in $A D$. Exposure to $A \beta$ alone is sufficient to cause progressive neuronal death. Although $A \beta$ is indeed neurotoxic by itself, the priming of neuronal inflammasomes, including NLRP1, by an external stimulus such as MDP significantly aggravates neuronal death as shown by the drastic decrease in neuronal viability in A $\beta+$ MDP treatment group. Interestingly, however, MDP by itself did not cause a significant change in cell viability, suggesting that inflammasome activation alone did not result in immediate cell death. It is also unlikely that $A \beta$ cause neuronal death via the NLRP1 inflammasome pathway as, by itself, it did not significantly elevate NLRP1 level, thus having insufficient caspase-1 activity to trigger pyroptosis (Fig. 3). However, when neurons were co-exposed with MDP, $A \beta$ was able to significantly elevate caspase- 1 activity, resulting in a drastic loss of neuronal viability. These results suggest that, while inflammasome and caspase-1 activation does not necessarily lead to neuronal death, co-stimulation with an external inflammagenic substance could lead inflammasome signalling into an overdrive sufficient to induce pyroptosis, thus significantly accelerating cell death. Therefore, the exposure of the brain to immunogenic stimuli such as pathogens, which are increasingly recognised for their roles in AD [22], could drastically accelerate neuronal death via inflammasome activation and pyroptosis. Indeed, it was proposed that $A \beta$ is deposited in the brain as part of an innate immune response against pathogenic infections [23].

The random genomic mutagenesis generated by gene trapping is a useful tool to screen for molecular mechanisms underlying a biological process. Unlike other gene silencing tools, such as siRNA, gene traps produce a permanent heterozygous (monoallelic) knockout which is equivalent to $50 \%$ reduction in gene product expression [11]. This is particularly advantageous as homozygous knockouts could be lethal, and 
human mutations are often heterozygous in nature. With FDR cut-off set to 0.05 , the gene trap mutagenesis phenotypic screen discovered seven significant genes which could highlight the underlying signalling pathways regulating neuronal survival and death when subjected to A $\beta$ and MDP-primed NLRP1 inflammasome. The most significant of these 'hit' genes are RHOT1 and CNP with mutant allele representation of log FC = 7.11 ('wild-type' susceptibility) and - 7.15 ('wild-type' protective) respectively in the $A \beta+$ MDP group. Both RHOT1 and $C N P$ has roles in mitochondrial toxicity, presumably a crucial factor in A $\beta$-induced NLRP1-mediated neuronal death. The RHOT1 gene encodes the Ras Homolog Family Member T1 protein, also known as Mitochondrial Rho GTPase 1 (Miro1). Miro1 is a member of the Rho family of GTPases, which in turn belongs to the Ras superfamily of small GTPases. Rho GTPases are typically small G proteins which are responsible for the regulation of many essential biological processes including cell growth, transcriptional regulation, membrane trafficking and cell motility $[24,25]$. Miro1 is essential in healthy neuronal function. Germline Miro1 knockout in mouse embryo die shortly after birth, and conditional knockout of neuronal Miro1 in a mouse model led to defects in mitochondria distribution and retrograde axonal mitochondrial movement, resulting in impaired motor neuron function [26]. However, Miro1 may also contribute to the pathogenesis of neurodegenerative diseases such as Parkinson's disease (PD) [27]. Miro1 is known to interact with the PD-associated proteins, parkin and PINK1, where Miro1 is phosphorylated by PINK1 and subsequently ubiquitinylated by parkin, leading to its degradation by proteasomes $[28,29]$. The degradation of Miro1 causes an arrest in mitochondria trafficking which quarantines defective mitochondria and prevents them from causing further damage to neighbouring healthy mitochondria. This immobilization of mitochondrial movement also facilitates its selective degradation via phagosomes and autolysosomes, a process known as mitophagy. Impaired Miro1 degradation disrupts mitophagic clearance of defective mitochondria which may elevate the production of reactive oxygen species (ROS) leading to the activation of the NLRP3 inflammasome $[30,31]$. Considering that Miro1-mediated neurotoxicity was only observed at a significant level in the A $\beta$ + MDP group, we hypothesize that A $\beta$ and the NLRP1

inflammasome could synergistically induce neuronal death through mitochondria dysfunction as in PD. It is possible that lack of Miro1 production in the library cells encourages mitophagic clearance of defective mitochondria and enabled cell survival.

Conversely, the CNP gene which encodes 2',3'-cyclic nucleotide 3' phosphodiesterase (CNPase) was found to be highly protective against $A \beta$-induced NLRP1-mediated neurotoxicity as indicated by a log FC $=-7.15$ for mutant $C N P$ in the $A \beta+$ MDP group. In the 2', $3^{\prime}$-cAMP-adenosine pathway, CNPase is found to convert 2',3'-cAMP to 2'-AMP which is further metabolized into adenosine [32]. Elevated adenosine confers neuroprotective effects against excitotoxic neuronal damage [33]. CNPase is also known to confer neuroprotection against mitochondria-induced toxicity. Specifically, CNPase regulates the opening of mitochondrial permeability transition pore (mPTP), a $\mathrm{Ca}^{2+}$-gated channel which is formed in mitochondria in response to $\mathrm{Ca}^{2+}$ overload or oxidative stress in order to increase the permeability of mitochondrial membrane [34,35]. Open mPTP abruptly increases the permeability of mitochondrial inner membrane (which is usually almost impermeable to solutes or ions) to molecules up to 1,500 Da, leading to excessive mitochondrial swelling and cell death by apoptosis or necrosis [36]. It is possible that 
mutation of $C N P$ gene encourages the opening of $\mathrm{MPTP}$ in response to $\mathrm{Ca}^{2+}$ and $\mathrm{ROS}$ imbalance in the cells as a result of $A \beta$ and MDP treatment.

The mutagenesis screen also identified two genes involved in TGF- $\beta$ signalling, TGFBR1 and SNW1. The TGFBR1 gene encodes the TGF- $\beta$ receptor type I (TGFBR1) protein which functions as a signal transduction component in TGF- $\beta$ signalling. TGFBR1 is integrated to the perpetually activated TGFBR2 as a dimer which spans the entire cell membrane. Upon binding to the extracellular ligands, principally TGF- $\beta$ cytokines, TGFBR1 is phosphorylated by TGFBR2, which in turn phosphorylates the intracellular SMAD family of proteins, specifically receptor-regulated SMAD proteins (R-SMADs) which consists of SMAD2 and SMAD3 [37]. Phosphorylated R-SMADs then accumulate into complexes with SMAD4 (a common SMAD or co-SMAD) which together act as transcription factors that signal the expression of target genes. As a negative regulatory mechanism for TGF- $\beta$ signalling, the transcription factor SKI acts as a co-repressor which binds to SMAD2-4, forming a complex which negatively regulates TGF- $\beta$ response [38]. The SKI-interacting protein (SKIP), encoded by the SNW1 gene, binds to SKI and antagonises its repressor effect, thereby releasing SMADs and allowing for their phosphorylation by TGFBR1, thus augmenting TGF- $\beta$ signalling [39]. Our functional screen shows that silencing TGFBR1 enabled neuronal survival in all three treatment groups at similar log FC in allele representation (mutant gene Log FC +3 , Table 1), suggesting that TGFBR1 confers susceptibility in response to toxic environment not specific to either $A \beta$ or MDP toxicity. SNW1, however, show an opposing effect in $A \beta$ and MDP groups with similar log FC (mutant gene Log $F C=-3$, Table 1) but not in the A + MDP group where there are no significant log FC compared to control, suggesting that activation of other pathways override the protective effects of $S N W 1$. TGF- $\beta$ signalling is a heterogenous signalling pathway that regulates many biological aspects such as cell proliferation and immunity [40]. The roles of TGF- $\beta$ signalling in neuroinflammation and $A D$ have been conflicting. It has been reported to promote neuroinflammation in $A D$ by encouraging the release of pro-inflammatory cytokines such as IL-1 $\beta$ and TNF- $\alpha$ in microvessels isolated in AD cortices [41] and blocking TGF- $\beta$ and its downstream SMAD2/3 signalling attenuates AD pathology [42]. Other studies indicate that TGF- $\beta$ is instead protective in nature, and its deficiency promotes neurodegeneration in AD [43]. Further studies are required to evaluate the impact of TGF- $\beta$ signalling, as well as its individual components, in neuroinflammation and AD pathogenesis.

Apart from TGFBR1 and SNW1 which constitute TGF- $\beta$ signalling, the functional screen identified $S R G A P 3$ as a protective gene unique to the $A \beta$ group. The SRGAP3 gene encodes the Slit-Robo Rho GTPase Activating Protein 3 (srGAP3), also known as WAVE-Associated Rac GTPase Activating Protein (WRP) or Mental Disorder-Associated GTPase Activating Protein (MEGAP), which belongs to the Rho GAP family of Rho GTPase negative regulators. srGAP3 is involved in a variety of processes which are crucial for neuronal morphogenesis, axon guidance and synaptic functions [44]. srGAP3 possibly confers neuroprotection against $A \beta$ toxicity by regulating cytoskeletal reorganisation or participating in Slit-Robo signalling, both of which are vital for neurodevelopment and healthy cognitive functions [45]. However, this neuroprotective effect is nullified in the presence of MDP, suggesting that the activation of NLRP1 inflammasome perturbs srGAP3 function and probably the regulation of cytoskeletal dynamics, which 
drastically reduced cellular rigidity against toxicity. This could have contributed to the synergism between $A \beta$ and MDP-primed NLRP1 inflammasome in inducing severe loss of neuronal viability in the A $\beta+$ MDP group.

$B E N D 5$ and GREB1 showed significance in the MDP group only, suggesting that exposure to $A \beta$ negated the effects exerted by these genes. As with SNW1, BEND5 is involved in DNA/RNA biding and transcription regulation. The BEND5 gene encodes the protein $\mathrm{BEN}$ domain-containing protein 5 . The mammalian BEND5 is a protein that contains a single BEN domain and is expressed in particularly high levels in pyramidal neurons [46]. It functions as a transcriptional repressor and is likely involved neurogenesis by regulating the Notch signalling pathway. The GREB1 gene codes for the growthregulating oestrogen receptor binding 1 protein. GREB1 is typically expressed upon binding of oestrogen response elements on oestrogen receptor 1 (ESR1) upstream of GREB1 promoter [47].Treatment with MDP alone did not result in significant loss of neuronal viability. It is possible that the effects of these mutant genes under MDP treatment affect cell proliferation rates rather than inducing neurodegeneration.

While the gene trap mutagenesis screening provides a convenient and high-throughput method for preliminary elucidation of biological pathways, screen efficiency is potentially limited by several factors. Firstly, the efficiency of gene trap integration is dependent on the initial nucleofection process. Secondly, although pGTIV3 does not display a 3 ' intron bias, its inherent requirement for intronic integration means that genes that lack, or contain very small, introns may escape trapping. This presents a major challenge in unbiased genome-wide mutagenesis by achieving complete genome saturation. Finally, the mutagenesis screen does not inform the order of gene activities and pathways involved - whether they occur upstream or downstream of NLRP1 inflammasome activation by A .

\section{Conclusion}

We have demonstrated that neuronal response to A $\beta$ is augmented when NLRP1 inflammasomes are primed by an external, immunogenic factor, suggesting that glia-independent neuroinflammation could result in significant neuronal self-destruction, likely via pyroptosis. Based on the genes identified by the mutagenesis screen, several putative pathways were identified which could be involved in regulating neuronal death in AD conditions, particularly in an NLRP1-primed state. We hypothesise that $A \beta$ and inflammasome could induce mitochondria toxicity similar to that observed in PD. Additionally, the mutagenesis screen was able to detect alterations in TGF- $\beta$ signalling which could play a role in neuroinflammation in AD. For future studies, it is recommended that these genes be targeted for knockout via other gene editing tools such as the CRISPR-cas9 system, or small molecule inhibitors where available, to validate the function of these genes. It is also imperative to test the effects of the identified genes and pathways in more complex models such as neuron-glia co-cultures or in vivo models, which would more accurately represent neuroinflammatory and AD conditions. The gene trap mutagenesis approach also presents a powerful tool in elucidating biological pathways and gene function especially if utilised in tandem with other gene expression or protein binding assessment techniques such as the GAL4/UAS system, which allows the study of specific gene function by targeted expression [48], and 
targeted DamID (TaDa), which offers sensitive and cell type-specific transcriptional profiling without requiring cell isolation, cell sorting, fixation, or affinity purification [49]. These techniques would be able to identify, in addition of novel signalling pathways, the genome-wide expression and interactions between genes in a particular pathway, which would undoubtedly prove to be highly advantageous for the identification and development of novel therapeutic targets for AD.

\section{Methods}

\section{$A \beta$ and MDP preparation}

Synthetic $A \beta_{1-42}$ peptide (Genscript, USA) was dissolved in dimethyl sulfoxide (DMSO) to a concentration of $5 \mathrm{mM}$ and further diluted to a $200 \mu \mathrm{M}$ stock solution with phosphate buffer saline (PBS). This solution was incubated at $4 \llbracket C$ for 24 hours and stored at $-20 \otimes C$ until use. During the experiment, the $A \beta$ was further diluted to a final concentration of $20 \mu \mathrm{M}$ in the samples. MDP (InvivoGen, USA) was prepared as a $10 \mathrm{mg} / \mathrm{mL}$ stock in endotoxin-free water. When used in experiments, the MDP was diluted to $100 \mu \mathrm{g} / \mathrm{mL}$ with serum-free media before further diluted in the samples to a final working concentration of $10 \mu \mathrm{g} / \mathrm{mL}$.

\section{Cell culture and treatments}

SH-SY5Y neuroblastoma cells (ATCC ${ }^{\circledR}$ CRL-2266 ${ }^{\mathrm{Tm}}$, American Type Culture Collection (ATCC), Virginia, USA) were cultured in T25 flasks with at least $5 \mathrm{~mL}$ of DMEM/F12 (1:1) culture media supplemented with GlutaMAX ${ }^{\mathrm{TM}}$ (Gibco, USA), 10\% foetal bovine serum (Biosera, France) and 1\% penicillin-streptomycin (Gibco, USA). The cells were incubated at $37 \llbracket \mathrm{C}$ with $5 \% \mathrm{CO}_{2}$ in humidified air. The culture medium was replaced every two days. At 70\% confluency, the cells were dissociated with TrypLE Express (Gibco, USA) and passaged in new T25 flasks. For cell treatment, serum-free cell culture media consisted of DMEM/F12 supplemented with GlutaMAX ${ }^{\mathrm{TM}}$ and $1 \%$ penicillin-streptomycin were used. Throughout this study, the treatment regimens were conducted in four groups: Control (cells exposed to only serum-free media), A $\beta$ (cells exposed to $20 \mu \mathrm{M} A \beta$ ), MDP (cells exposed to $10 \mu \mathrm{g} / \mathrm{mL} \mathrm{MDP}$ ) and A + MDP (cells coexposed to $20 \mu \mathrm{M} \mathrm{A} \beta$ and $10 \mu \mathrm{g} / \mathrm{mL} \mathrm{MDP})$.

\section{Cell viability assay}

Cell viability was measured using the RealTime-Glo MTTM Cell Viability Assay kit (Promega, USA). SHSY5Y cells were seeded at the density of $3 \times 10^{4}$ cells/well in a white opaque 96-well plate and incubated overnight. The serum-free media, MT Cell Viability Substrate and NanoLuc $®$ Enzyme (components of the assay kit) were pre-warmed in a $37 \mathbb{C}$ water bath prior to preparation of a $2 x$ concentration of enzymesubstrate mixture. The mixture was then added into the cell culture samples to a final concentration of 1x. Cell treatment was conducted as described for the four treatment groups. The luminescence signal was measured with SpectraMAX M3 microplate reader at 2-hour time points for 72 hours. The assay was performed with six replicates.

\section{Western blot}


For protein quantification, SH-SY5Y cells were seeded in 6-well plates $\left(2 \times 10^{6}\right.$ cells/well) and allowed to stabilize for 24 hours. The cells were then treated as described for 2 hours. Total protein in the cell lysates were harvested in RIPA buffer (Abcam, UK) and quantified using the Bradford assay (Bio-Rad, USA). Each well was loaded with $30 \mu \mathrm{g}$ protein in Laemmli buffer (Bio-Rad, USA). Proteins were separated using 10\% sodium dodecyl sulphate polyacrylamide (SDS-PAGE) gel at 120V and blotted onto a polyvinylidene difluoride (PVDF) membrane. The membrane was then blocked with Blocking One (Nacalai Tesque, Japan) for one hour at room temperature. The proteins of interest in each sample were probed using primary antibodies (anti-NLRP1, anti-caspase-1 and anti- $\beta$-actin; Cell signalling Technology, USA) at 1:1000 dilution. $\beta$-actin was used as loading control. Anti-rabbit IgG HRP-conjugated antibody (Cell Signalling Technology, Massachusetts, USA) at 1:2000 was used as secondary antibody. The ChemiLumi One Super enhanced chemiluminescence reagent (Nacalai Tesque, Japan) was used to detect the blots. Blot visualization and densitometric analyses were performed using Bio-Rad Image Lab 6.0.1 software. All western blots were performed in triplicates.

\section{Statistical analyses}

The differences of means between the treatment groups were analysed with one-way analysis of variance (ANOVA) followed by Tukey's Honest Significant Difference post-hoc test. All statistical analyses were computed with GraphPad Prism 8.0.2 software.

\section{Gene-trap mutagenesis and selection process}

The pGTIV3 plasmid was linearized using the Pvul restriction enzyme and then transfected into SH-SY5Y cells via nucleofection utilizing Cell Line Nucleofector ${ }^{\mathrm{TM}}$ Kit V (Lonza, Switzerland), followed by G418/Geneticin selection for productive mutation events, and expansion to produce multiple aliquots. The resulting library cells were seeded in T12.5 flasks $\left(5 \times 10^{5}\right.$ cells/flask), incubated overnight and subjected to the various treatments as described for 72 hours. Surviving populations of cells were allowed to recover to full confluency before being re-seeded (excess cells archived as frozen stocks) and re-treated for another 72 hours. This treatment-recovery process was performed for 7 cycles.

\section{First strand cDNA synthesis and RACE}

At the end of the 7 cycles of selection process, the surviving cells were allowed to recover in T75 flasks. At full confluency, the total RNA was extracted from these cells with ISOLATE II RNA Mini Kit (Bioline, UK) according to the manufacturer's protocol. From the eluted RNA, first strand CDNA was synthesized using SuperScript IV Reverse Transcriptase (Invitrogen, USA). The modified oligo(dT) primer used for reverse transcription (3CDSynth) was 5' AAG CAG TGG TAA CAA CGC AGA GTA CTT TTT TTT TTT TTT TTT TTT TTT TTT TTT TVN 3'.

We utilized the 3' RACE method to amplify neoR- mutant allele fusion transcripts in the experimental and control groups for identification and quantification purposes. This takes advantage of the natural poly(A) tail as a functional priming site to attach an adaptor via the modified oligo(dT) primer and produce a first strand cDNA with a known sequence (neoR) at the 5 ' end. To ensure the specificity of the final amplicons, 
the cDNA strands were amplified through a series of nested PCR steps utilizing two sets of forward and reverse primers (RACE\#1 and RACE\#2 primers). RACE\#1 primers consist of SD5-P1 (forward) 5' GCT TGC CGA ATA TCA TGG TGG AAA ATG G 3' and UPM (reverse) (UPM is a mixture of $0.4 \mu \mathrm{M}$ UPL 5' CTA ATA CGA CTC ACT ATA GGG CAA GCA GTG GTA ACA ACG CAG AGT 3' + $2 \mu$ M UPS 5' CTA ATA CGA CTC ACT ATA GGG C 3'). RACE\#2 primers are SD5-P3 (forward) 5' CGC ATC GCC TTC TAT CGC CTT CTT GAC G 3' and Nest1 (reverse) 5' AAG CAG TGG TAA CAA CGC AGA GT 3'. The thermal cycling parameters set were:

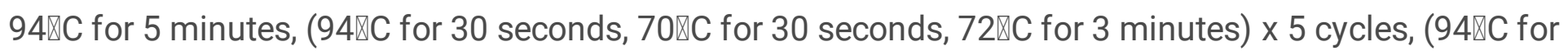
30 seconds, $68 \otimes C$ for 30 seconds, $72 \bowtie C$ for 3 minutes) $\times 30$ cycles, and finally $72 \varangle \mathrm{C}$ for 5 minutes.

\section{Library preparation and next generation sequencing}

The amplicons were taken through library construction using the Illumina TruSeq Nano Library Prep kit. Paired-end targeted sequencing was performed with the Illumina NextSeq instrument which produced $75 \mathrm{bp}$ reads for each end, totalling 30 million reads. Only the subset of transcripts produced by the gene trap insertional mutagenesis (neomycin resistance - mutated gene fusions) were sequenced. Because of the reduced genomic complexity, we opted to perform multiplex sequencing by incorporating 'barcode' sequences to each DNA fragment during library preparation to distinguish between samples and replicates within the amplicon pool $(n=3)$.

\section{Bioinformatics analysis}

NGS data analysis was conducted with the repository of Bioconductor tools available as GUI at the webbased Galaxy platform (https://usegalaxy.org/). The NGS data were available in FASTQ format compressed in gz files (fastq.gz). The FASTQ files were converted into FASTQ Sanger format (fastqsanger.gz) using the FASTQ Groomer tool in order to ensure compatibility with the Galaxy platform [16]. The raw sequences were then mapped into the human genome (hg38) using the HISAT2 aligner with default parameters to produce BAM files outputs [17]. The aligned sequences were then assembled into transcripts using StringTie transcript assembler [18]. An Ensembl hg38 gene annotation file was used to guide transcript assembly and only transcripts that matched the reference annotation file were accepted (The parameter 'Use Reference transcripts only?' was set to True). The assembler was also set to output a tabular file containing gene counts compatible with the limma-voom software package. Limma-voom analysis and data visualization were performed on the Degust online platform (http://degust.erc.monash.edu/) [19].

\section{Abbreviations}

$A D$

Alzheimer's disease

CNS

Central nervous system

$A \beta$

Amyloid- $\beta$ 
MDP

Muramyl dipeptide

NLRP1

Nucleotide-binding oligomerization Domain, leucine rich repeat and pyrin domain-containing 1 NLRP3

Nucleotide-binding oligomerization Domain, leucine rich repeat and pyrin domain-containing 3 SD

Splice donor

ARE

$\mathrm{AU}$ rich element

RACE

Rapid amplification of cDNA ends

NGS

Next-generation sequencing

Log FC

$\log _{2}$ fold change

PD

Parkinson's disease

\section{Declarations}

\section{Ethics approval and consent to participate}

Not applicable

\section{Consent for publication}

Not applicable

\section{Data availability statement}

The datasets used and/or analysed during the current study are available from the corresponding author on reasonable request.

\section{Competing interests}

The authors declare that they have no competing interests.

\section{Funding}

This work is funded by the Fundamental Research Grant Scheme, Ministry of Higher Education of Malaysia (FRGS/1/2016/SKK08/IMU/03/1). 


\section{Authors' Contributions}

EWLC, SYG and BSP designed the study. JKYY and BSP performed the experiments. JKYY, EWLC and BSP analysed the data. JKYY wrote the manuscript. EWLC, SYG, BSP and JKYY contributed to the final edition of the manuscript.

\section{Acknowledgment}

We would like to thank Glasgow Polyomics, University of Glasgow for their services in library preparation and NGS.

\section{References}

1. Masters CL, Bateman R, Blennow K, Rowe CC, Sperling RA, Cummings JL. Alzheimer's disease. Nat Rev Dis Prim. 2015;1:15056. doi:10.1038/nrdp.2015.56.

2. Kinney JW, Bemiller SM, Murtishaw AS, Leisgang AM, Salazar AM, Lamb BT. Inflammation as a central mechanism in Alzheimer's disease. Alzheimer's Dement Transl Res Clin Interv. 2018;4:57590. doi:10.1016/j.trci.2018.06.014.

3. Sarlus H, Heneka MT. Microglia in Alzheimer's disease. J Clin Invest. 2017;127:33-5.

4. Yap JKY, Pickard BS, Chan EWL, Gan SY. The Role of Neuronal NLRP1 Inflammasome in Alzheimer's Disease: Bringing Neurons into the Neuroinflammation Game. Mol Neurobiol. 2019;56:7741-53.

5. Bergsbaken T, Fink SL, Cookson BT. Pyroptosis: Host cell death and inflammation. Nat Rev Microbiol. 2009;7:99-109.

6. Tan M-S, Yu J-T, Jiang T, Zhu X-C, Tan L. The NLRP3 Inflammasome in Alzheimer's Disease. Mol Neurobiol. 2013;48:875-82. doi:10.1007/s12035-013-8475-x.

7. Tan M-S, Tan L, Jiang T, Zhu X-C, Wang H-F, Jia C-D, et al. Amyloid- $\beta$ induces NLRP1-dependent neuronal pyroptosis in models of Alzheimer's disease. Cell Death Dis. 2014;5:e1382. doi:10.1038/cddis.2014.348.

8. Walsh JG, Muruve DA, Power C. Inflammasomes in the CNS. Nat Rev Neurosci. 2014;15:84-97. doi:10.1038/nrn3638.

9. Kaushal V, Dye R, Pakavathkumar P, Foveau B, Flores J, Hyman B, et al. Neuronal NLRP1 inflammasome activation of Caspase-1 coordinately regulates inflammatory interleukin-1-beta production and axonal degeneration-associated Caspase- 6 activation. Cell Death Differ. 2015;22:1676-86. doi:10.1038/cdd.2015.16.

10. Salminen M, Meyer BI, Gruss P. Efficient poly a trap approach allows the capture of genes specifically active in differentiated embryonic stem cells and in mouse embryos. Dev Dyn. 1998;212:326-33.

11. Gow M, Mirembe D, Longwe Z, Pickard BS. A gene trap mutagenesis screen for genes underlying cellular response to the mood stabilizer lithium. J Cell Mol Med. 2013;17:657-63. 
12. Morris SM, Mhyre AJ, Carmack SS, Myers $\mathrm{CH}$, Burns C, Ye W, et al. A modified gene trap approach for improved high-throughput cancer drug discovery. Oncogene. 2018;37:4226-38. doi:10.1038/s41388018-0274-4.

13. Tsakiridis A, Tzouanacou E, Rahman A, Colby D, Axton R, Chambers I, et al. Expression-independent gene trap vectors for random and targeted mutagenesis in embryonic stem cells. Nucleic Acids Res. 2009;37:e129-9.

14. Faustin B, Lartigue L, Bruey JM, Luciano F, Sergienko E, Bailly-Maitre B, et al. Reconstituted NALP1 Inflammasome Reveals Two-Step Mechanism of Caspase-1 Activation. Mol Cell. 2007;25:713-24.

15. Hsu LC, Ali SR, McGillivray S, Tseng PH, Mariathasan S, Humke EW, et al. A NOD2-NALP1 complex mediates caspase-1-dependent IL-1 $\beta$ secretion in response to Bacillus anthracis infection and muramyl dipeptide. Proc Natl Acad Sci U S A. 2008.

16. Blankenberg D, Gordon A, Von Kuster G, Coraor N, Taylor J, Nekrutenko A, et al. Manipulation of FASTQ data with galaxy. Bioinformatics. 2010.

17. Kim D, Langmead B, Salzberg SL. HISAT: A fast spliced aligner with low memory requirements. Nat Methods. 2015.

18. Pertea M, Pertea GM, Antonescu CM, Chang TC, Mendell JT, Salzberg SL. StringTie enables improved reconstruction of a transcriptome from RNA-seq reads. Nat Biotechnol. 2015.

19. Law CW, Chen Y, Shi W, Smyth GK. voom: Precision weights unlock linear model analysis tools for RNA-seq read counts. Genome Biol. 2014.

20. Shi CS, Shenderov K, Huang NN, Kabat J, Abu-Asab M, Fitzgerald KA, et al. Activation of autophagy by inflammatory signals limits IL-1 $\beta$ production by targeting ubiquitinated inflammasomes for destruction. Nat Immunol. 2012;13:255-63.

21. Sun Q, Fan J, Billiar TR, Scott MJ. Inflammasome and autophagy regulation - a two-way street. Mol Med. 2017;23:188-95.

22. Miklossy J. Emerging roles of pathogens in Alzheimer disease. Expert Rev Mol Med. 2011;13:e30.

23. Moir RD, Lathe R, Tanzi RE. The antimicrobial protection hypothesis of Alzheimer's disease. Alzheimer's Dement. 2018;14:1602-14. doi:10.1016/j.jalz.2018.06.3040.

24. Aznar S, Lacal JC. Rho signals to cell growth and apoptosis. Cancer Lett. 2001;165:1-10.

25. Hanna S, El-Sibai M. Signaling networks of Rho GTPases in cell motility. Cell Signal. 2013;25:195561. doi:10.1016/j.cellsig.2013.04.009.

26. Nguyen TT, Oh SS, Weaver D, Lewandowska A, Maxfield D, Schuler M-H, et al. Loss of Miro1-directed mitochondrial movement results in a novel murine model for neuron disease. Proc Natl Acad Sci. 2014.

27. Devine MJ, Birsa N, Kittler JT. Miro sculpts mitochondrial dynamics in neuronal health and disease. Neurobiol Dis. 2016;90:27-34.

28. van der Merwe C, Jalali Sefid Dashti Z, Christoffels A, Loos B, Bardien S. Evidence for a common biological pathway linking three Parkinson's disease-causing genes: Parkin, PINK1 and DJ-1. Eur J 
Neurosci. 2015;41:1113-25.

29. Hsieh C-H, Shaltouki A, Gonzalez AE, Bettencourt da Cruz A, Burbulla LF, St. Lawrence E, et al. Functional Impairment in Miro Degradation and Mitophagy Is a Shared Feature in Familial and Sporadic Parkinson's Disease. Cell Stem Cell. 2016;19:709-24. doi:10.1016/j.stem.2016.08.002.

30. Gao J, Sang M, Zhang X, Zheng T, Pan J, Dai M, et al. Miro1-mediated mitochondrial dysfunction under high nutrient stress is linked to NOD-like receptor 3 (NLRP3)-dependent inflammatory responses in rat pancreatic beta cells. Free Radic Biol Med. 2015;89:322-32. doi:10.1016/j.freeradbiomed.2015.09.002.

31. Chen L, Liu C, Gao J, Xie Z, Chan LWC, Keating DJ, et al. Inhibition of Miro1 disturbs mitophagy and pancreatic $\beta$-cell function interfering insulin release via IRS-Akt-Foxo1 in diabetes. Oncotarget. 2017;8.

32. Jackson EK. The 2',3'-cAMp-adenosine pathway. American Journal of Physiology - Renal Physiology. 2011.

33. Wardas J. Neuroprotective role of adenosine in the CNS. Polish Journal of Pharmacology. 2002.

34. Krestinina O, Azarashvili T, Baburina Y, Galvita A, Grachev D, Stricker R, et al. In aging, the vulnerability of rat brain mitochondria is enhanced due to reduced level of 2',3'-cyclic nucleotide-3'-

phosphodiesterase (CNP) and subsequently increased permeability transition in brain mitochondria in old animals. Neurochem Int. 2015.

35. Baburina Y, Azarashvili T, Grachev D, Krestinina O, Galvita A, Stricker R, et al. Mitochondrial 2', 3'cyclic nucleotide 3'-phosphodiesterase (CNP) interacts with MPTP modulators and functional complexes (I-V) coupled with release of apoptotic factors. Neurochem Int. 2015.

36. Lemasters JJ, Theruvath TP, Zhong Z, Nieminen A. Mitochondrial calcium and the permeability transition in cell death. BBA - Bioenerg. 2009;1787:1395-401. doi:10.1016/j.bbabio.2009.06.009.

37. Hata A, Chen YG. TGF- $\beta$ signaling from receptors to smads. Cold Spring Harbor Perspectives in Biology. 2016;8.

38. Xu W, Angelis K, Danielpour D, Haddad MM, Bischof O, Campisi J, et al. Ski acts as a co-repressor with Smad2 and Smad3 to regulate the response to type $\beta$ transforming growth factor. Proc Natl Acad Sci U S A. 2000.

39. Leong GM, Subramaniam N, Figueroa J, Flanagan JL, Hayman MJ, Eisman JA, et al. Ski-interacting Protein Interacts with Smad Proteins to Augment Transforming Growth Factor- $\beta$-dependent Transcription. J Biol Chem. 2001.

40. Batlle E, Massagué J. Transforming Growth Factor- $\beta$ Signaling in Immunity and Cancer. Immunity. 2019;50:924-40.

41. Grammas P, Ovase R. Cerebrovascular transforming growth factor- $\beta$ contributes to inflammation in the Alzheimer's disease brain. Am J Pathol. 2002;160:1583-7.

42. Town T, Laouar Y, Pittenger C, Mori T, Szekely CA, Tan J, et al. Blocking TGF- $\beta$-Smad $2 / 3$ innate immune signaling mitigates Alzheimer-like pathology. Nat Med. 2008;14:681-7. 
43. Tesseur I, Zou K, Esposito L, Bard F, Berber E, Van Can J, et al. Deficiency in neuronal TGF- $\beta$ signaling promotes neurodegeneration and Alzheimer's pathology. J Clin Invest. 2006.

44. Huang GH, Sun ZL, Li HJ, Feng DF. Rho GTPase-activating proteins: Regulators of Rho GTPase activity in neuronal development and CNS diseases. Mol Cell Neurosci. 2017;80:18-31. doi:10.1016/j.mcn.2017.01.007.

45. Bacon C, Endris V, Rappold GA. The cellular function of srGAP3 and its role in neuronal morphogenesis. Mech Dev. 2013;130:391-5. doi:10.1016/j.mod.2012.10.005.

46. Dai Q, Ren A, Westholm JO, Serganov AA, Patel DJ, Lai EC. The BEN domain is a novel sequencespecific DNA-binding domain conserved in neural transcriptional repressors. Genes Dev. 2013;27:602-14.

47. Sun J, Nawaz Z, Slingerland JM. Long-Range Activation of GREB1 by Estrogen Receptor via Three Distal Consensus Estrogen-Responsive Elements in Breast Cancer Cells. Mol Endocrinol. 2007;21:2651-62.

48. Brand AH, Perrimon N. Targeted gene expression as a means of altering cell fates and generating dominant phenotypes. Development. 1993;118:401-15.

49. Southall TD, Gold KS, Egger B, Davidson CM, Caygill EE, Marshall OJ, et al. Cell-type-specific profiling of gene expression and chromatin binding without cell isolation: Assaying RNA pol II occupancy in neural stem cells. Dev Cell. 2013;26:101-12. doi:10.1016/j.devcel.2013.05.020.

\section{Figures}




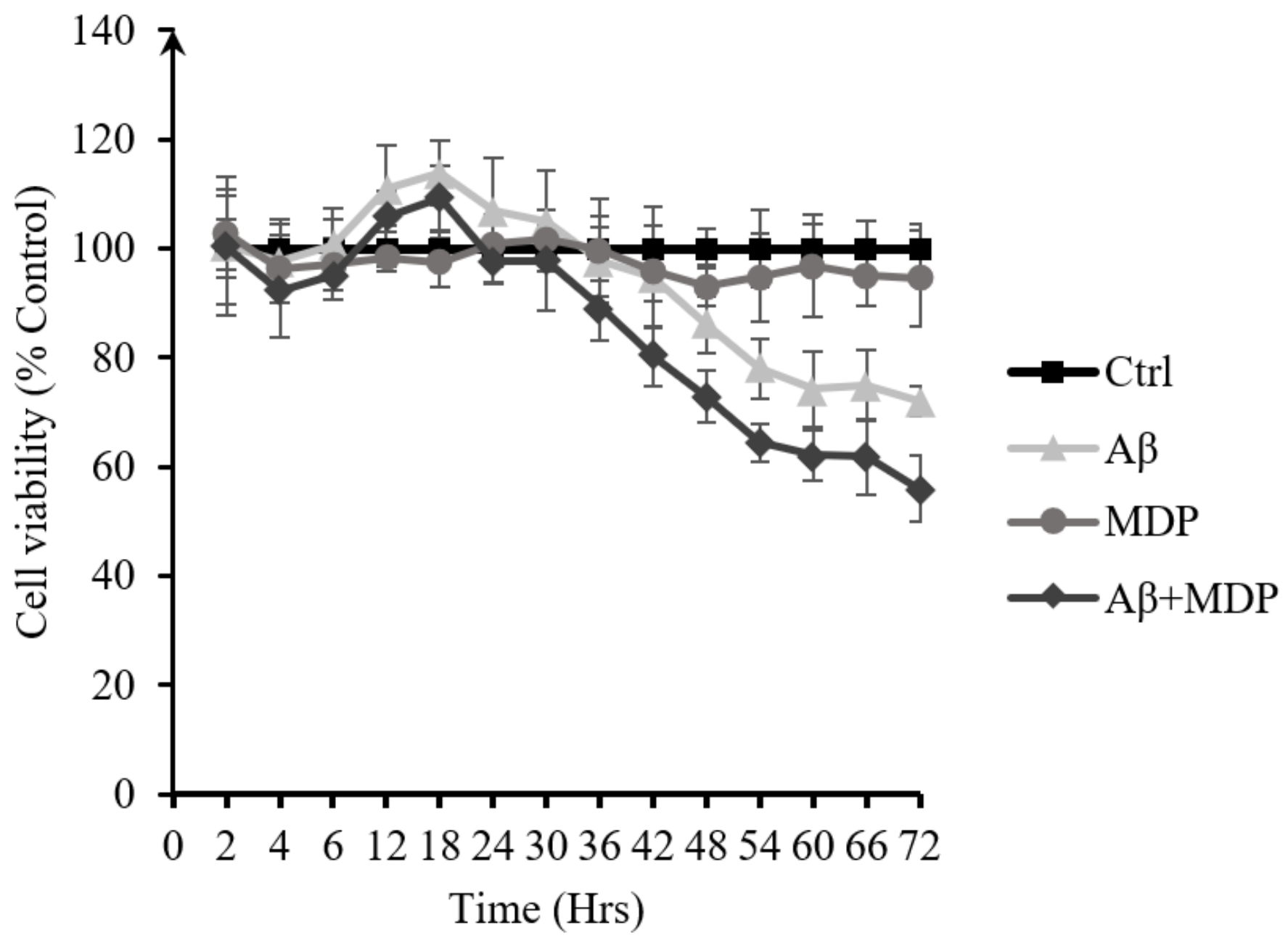

Figure 1

SHSY-5Y cell viability in each treatment group compared to control, measured in real time over 72 hours. To allow the assay to stabilise, the luminescent signal was measured after two hours after addition of reagents. Data are presented as mean $\pm \operatorname{SD}(n=6)$. 


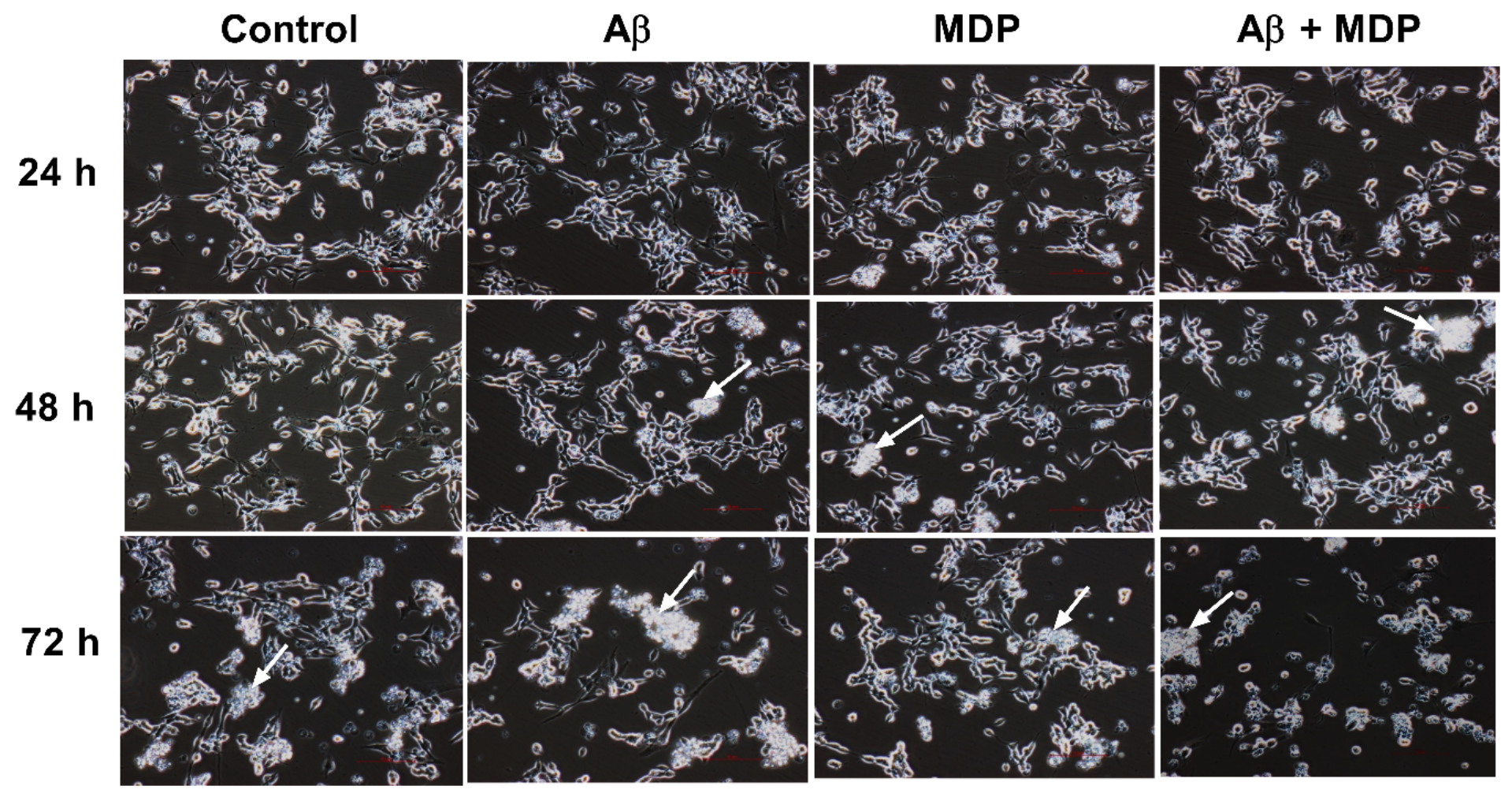

Figure 2

Phase-contrast microscopic images of SH-SY5Y cells in each treatment groups over 72 hours. Arrow indicates clumps of cell lesions. Images are captured at 200x magnification ( $n=3,3$ fields are taken for each sample). 
(A)

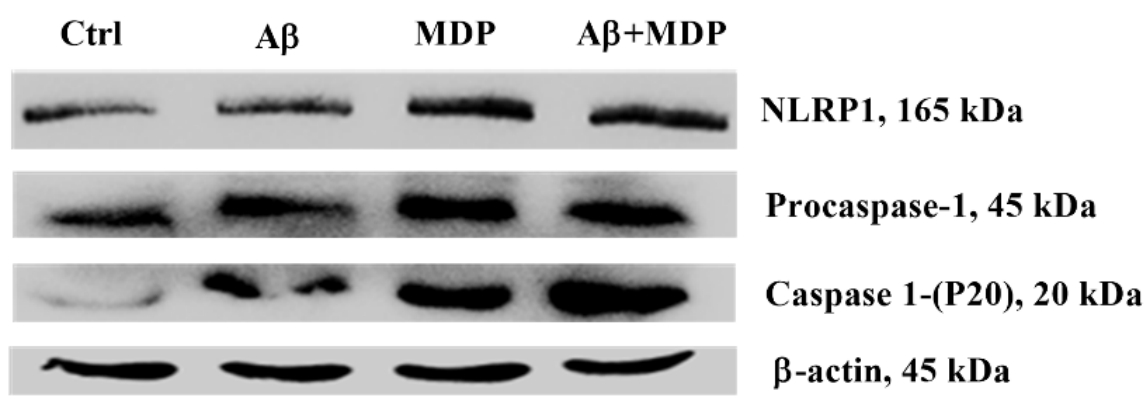

(B)
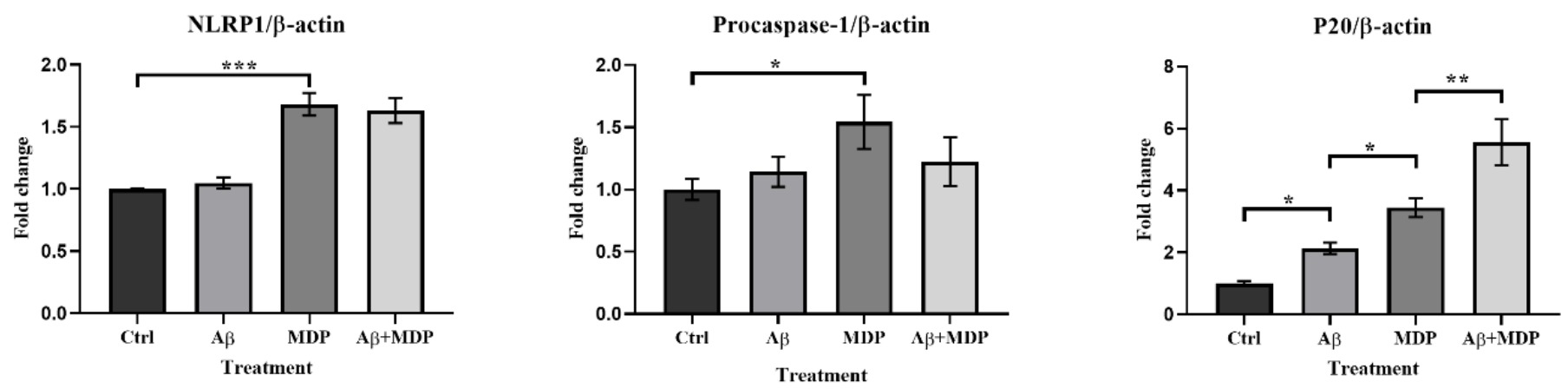

Figure 3

(A) Western blot of NLRP1 and caspase-1 normalized against $\beta$-actin. The proteins were quantified from total protein harvested from the cell lysates. (B) Corresponding densitometric analyses. The data are presented as mean $\pm S D(n=3)$. * denotes $P<0.05$, ** denotes $P<0.005$, *** denotes $P<0.0005$.

(A)

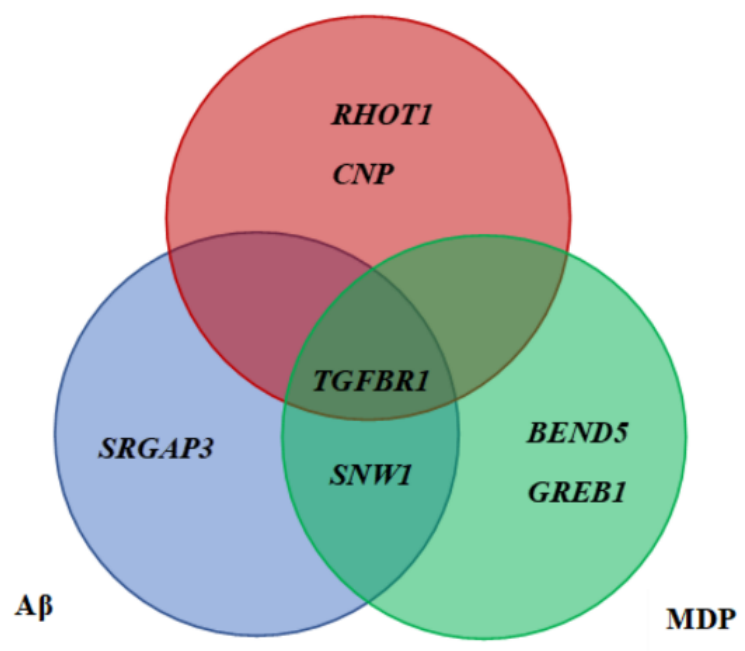

(B)

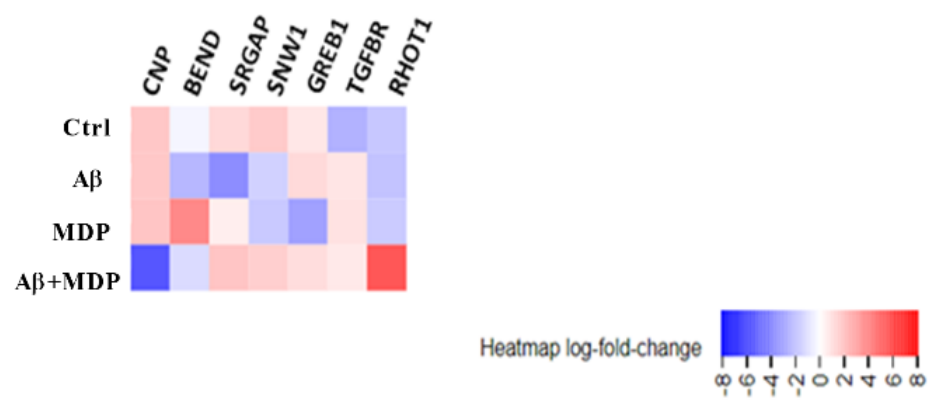

Figure 4 

(A) Venn diagram depicting the significant genes (FDR cut-off $=0.05$ ) contributing to neuronal survival or death in each treatment group compared to control. The overlapping sections indicate the genes common to multiple pair-wise comparisons between groups. (B) Heatmap representative of changes in allele frequency of significant 'hit' genes in surviving mutant cell population across treatment groups. 